\title{
Hybrid Digital-Analog Coding of Memoryless Gaussian Sources over AWGN Channels with Bandwidth Compression*
}

\author{
Yadong Wang \\ Dept. of Mathematics \& Statistics \\ Queen's University \\ Kingston, ON, K7L 3N6, Canada. \\ Email: yadong@mast.queensu.ca
}

\author{
Fady Alajaji \\ Dept. of Mathematics \& Statistics \\ Queen's University \\ Kingston, ON, K7L 3N6, Canada. \\ Email: fady@mast.queensu.ca
}

\author{
Tamás Linder \\ Dept. of Mathematics \& Statistics \\ Queen's University \\ Kingston, ON, K7L 3N6, Canada. \\ Email: linder@mast.queensu.ca
}

\begin{abstract}
We present a low-complexity and low-delay joint source-channel coding method for bandwidth compression using a hybrid digital-analog (HDA) coding/modulation system based on the recent work in [3]. Analytical optimal distortion expressions (under both matched and mismatched channel conditions) are obtained for the proposed HDA system with a linear analog part for a memoryless Gaussian source and additive white Gaussian noise (AWGN) channel under the mean squared error distortion measure. We consider two HDA coding schemes, both of which employ a vector quantizer cascaded with binary phaseshift keying (BPSK) modulation in the digital part, but differ in that they use linear (resp. non-linear) coding with pulse amplitude modulation (PAM) in the analog part. We derive an optimal power allocation scheme for the system with linear analog coding and present performance comparisons with purely analog and purely digital systems, as well as the scheme in [3]. Simulation results show that, under linear analog coding, the proposed scheme outperforms the scheme of [3] for medium to high channel signal-to-noise ratios (CSNRs). Furthermore, the performance of the HDA scheme with the linear analog part is within $1 \mathrm{~dB}$ of the optimal distortion bound for the mismatched HDA system for high CSNRs; for the scheme with non-linear analog coding, the performance can be improved at high CSNRs.
\end{abstract}

Index Terms: Hybrid digital-analog coding, joint source-channel coding, vector quantization, broadcasting, robustness.

\section{INTRODUCTION}

We consider the problem of transmitting analog-valued sources over a discrete-time memoryless channel. Due to the usual lack of channel information at the transmitter, a robust system is desirable. In terms of the used modulation techniques, systems can be categorized into analog, digital or hybrid digital-analog (HDA). Digital systems have the advantage that they can be designed to (asymptotically) achieve the theoretical optimal performance for a fixed channel signal-tonoise ratio (CSNR) via the separate design of optimal source and channel codes. There are, however, two fundamental disadvantages associated with digital tandem systems. One is the threshold effect: the system typically performs well at the design CSNR, while it degrades drastically when the true CSNR falls beneath the design CSNR. This effect is due to the quantizer's sensitivity to channel errors and the breakdown

\footnotetext{
${ }^{*}$ This work was supported in part by NSERC of Canada and PREA of
} Ontario. of the employed error-correcting code at low CSNRs (no matter how powerful it is). The other trait is the levellingoff effect: as the CSNR increases, the performance remains constant beyond a certain threshold. This is due to the nonrecoverable distortion introduced by the quantizer which limits the system performance at high CSNRs. The threshold effect can be partly remedied by digital joint source-channel coding (JSCC) design. However, such JSCC systems still suffer from the levelling-off effect at high CSNRs, since being digital systems, they employ quantization to "digitize" the source. On the other hand, the levelling-off effect is not a problem for analog systems, which means that the performance of an analog system can strictly increase as the CSNR increases. However, it is usually hard to incorporate efficient signal compression schemes in analog systems, particularly for sources with memory and when channel bandwidth is valuable.

Recent works undertaken to exploit the advantages of both analog and digital systems are as follows. In [1], a family of HDA systems are introduced and studied theoretically; they are shown to offer better distortion performance than the purely digital system, have a less severe threshold effect, and (asymptotically) achieve the Shannon limit. An HDA system design based on vector quantization (VQ) for bandwidth expansion is investigated in [2], where an algorithm to design optimized codes and distortion performance are presented. In [3], an HDA system for Gauss-Markov sources with bandwidth compression/expansion is presented. It employs the Karhunen-Loéve transform to decorrelate the source, Turbo error-correcting coding in its digital part to improve the system performance at low CSNRs, and superposition coding of the analog and digital signals. This system allows for both linear and non-linear mapping in its analog component. Other related HDA results can be found in [4] - [14].

In this work, we study the transmission of a memoryless Gaussian source over an additive white Gaussian noise (AWGN) channel with bandwidth compression, using HDA techniques based on the recent work in [3]. We first obtain a theoretical distortion bound of an HDA system with a linear analog part for the mean squared error (MSE) distortion measure. Then we derive a theoretical distortion bound of a mismatched HDA system where the encoder does not know the true CSNR. An optimal power allocation formula between the 


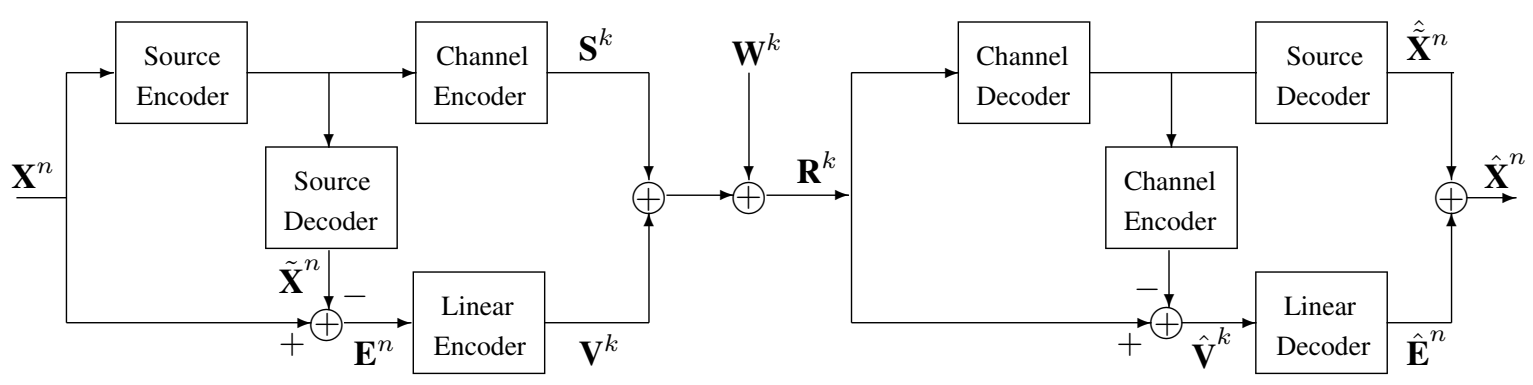

Fig. 1. A general HDA model with bandwidth compression $(k<n)$.

digital and the analog parts is obtained for this mismatched system. Using this formula, a power allocation that is good over a wide range of CSNRs is chosen to design an HDA scheme. This HDA scheme (which, unlike [3], does not employ Turbo codes) has low complexity and low delay. The digital part is formed with a VQ (optimized as in [5]) cascaded with a binary phase shift keying (BPSK) modulated harddecision decoded AWGN channel, and the analog part uses linear coding. Simulations show that this scheme performs within $1 \mathrm{~dB}$ of the optimal performance of the mismatched HDA system for high CSNRs. As the performance saturates with increased CSNR, a non-linear analog coding is also considered in the proposed HDA scheme to improve the saturation level. Comparison are made with purely analog and purely digital systems, as well as the system in [3].

\section{INFORMATION-THEORETIC CONSIDERATIONS}

The block diagram for the HDA system with bandwidth compression is depicted in Fig.1. The samples of a memoryless Gaussian source $\left\{X_{i}\right\}$ with zero mean and variance $\sigma_{s}^{2}$ are grouped into blocks of size $n$, denoted as $\mathbf{X}^{n}$, and encoded by a digital tandem source-channel encoder with power $(1-t) P$, where $P$ is the total input power per channel use and $t \in[0,1]$ is the power allocation coefficient for the analog part. The source decoder output $\tilde{\mathbf{X}}^{n}$ is subtracted from $\mathbf{X}^{n}$ to form the error vector $\mathbf{E}^{n}, k$ components of which are further transmitted using linear analog coding (via a scaling operation) with power constraint $E\left\|\mathbf{V}^{k}\right\|^{2} \leq k t P$. $\mathbf{S}^{k}$ and $\mathbf{V}^{k}$ are superposed and sent over an AWGN channel with noise variance $N$. The receiver first employs a digital tandem decoder to get an estimate $\hat{\mathbf{X}}^{n}$. The decoded channel symbols are subtracted from $\mathbf{R}^{k}$. The results are fed to the linear analog decoder to form an estimate $\hat{\mathbf{E}}^{n}$. Here, the remaining $n-k$ components of the error vector are filled with zeros at the decoder part. $\hat{\mathbf{E}}^{n}$ is then added to $\hat{\tilde{\mathbf{X}}}^{n}$ to form an estimate $\hat{\mathbf{X}}^{n}$. The overall coding rate of this HDA system is $r=k / n<1$ channel uses per source sample.

We first consider the optimal asymptotic distortion of this system

$$
D_{M S E}(N)=\limsup _{n \rightarrow \infty} \frac{1}{n} E\|\mathbf{X}-\hat{\mathbf{X}}\|^{2} .
$$

From Shannon's lossy JSCC theorem [15], we know that the performance of a general JSCC system for sending an i.i.d.
Gaussian source over an AWGN channel is always lower bounded by the optimal performance theoretically attainable (OPTA), which is

$$
D_{O P T A}(N) \triangleq \frac{\sigma_{s}^{2}}{\left(1+\frac{P}{N}\right)^{r}} .
$$

By examining the structure for the proposed HDA system in Fig.1, we first derive an OPTA for this system.

Lemma 1: For a memoryless Gaussian source with zero mean and variance $\sigma_{s}^{2}$ and an AWGN channel with noise variance $N$, given fixed $P, t$ and $r$, the OPTA of the HDA system of Fig.1, denoted by $D_{O P T A}^{h d a}(N)$, is given by

$$
D_{O P T A}^{h d a}(N) \triangleq\left(\frac{r}{1+\frac{t P}{N}}+(1-r)\right) D_{d i g}(N),
$$

where

$$
D_{d i g}(N) \triangleq \frac{\sigma_{s}^{2}}{\left(1+\frac{(1-t) P}{t P+N}\right)^{r}} .
$$

It is easy to show that $D_{O P T A}^{h d a}(N)=D_{O P T A}(N)$ if and only if $t=0$.

We next study the realistic situation where the AWGN variance $N$ is not known at the encoder. We assume that the encoder only knows a range of values in which the true noise variance $N$ lies; thus, it chooses the encoding operation for a fixed design noise variance $N_{\text {des }}$. The receiver on the other hand has full knowledge of $N$ and adapts the decoding accordingly. For this mismatched HDA system, when the true noise variance $N$ satisfies $N \leq N_{\text {des }}$, we have the following OPTA distortion:

$D_{O P T A}^{m i s-h d a}\left(N, N_{d e s}\right) \triangleq\left(\frac{r}{1+\frac{t P}{N}}+(1-r)\right) D_{d i g}\left(N_{d e s}\right)$.

We now consider the power allocation problem for this mismatched HDA system with the encoder designed for $N_{\text {des }}$, while the true noise variance is $N$. The best power allocation coefficient $t$ that minimizes (5) is given by the following lemma.

Lemma 2: For a given $N_{\text {des }} \geq N, P$ and $r$, the best power allocation coefficient $t$ which minimizes the distortion 


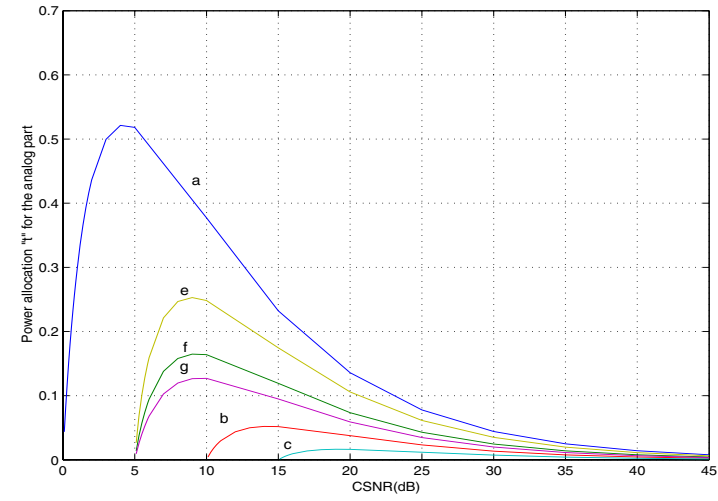

Fig. 2. The best power allocation $t$ (shown by the y-coordinate) for different system parameters. For curves (a), (b) and (c), $r=0.5, \kappa_{d}=1,10$ and 32 respectively. For curves (e), (f) and (g), $\kappa_{d}=3.2, r=0.75,0.5$ and 0.25 respectively. CSNR values are shown (in $\mathrm{dB}$ ) by the $\mathrm{x}$-coordinate.

expression (5) at $N$ is given by

$$
t=\frac{\sqrt{1+\frac{4\left(\kappa-\kappa_{d}\right)}{(1-r) \kappa_{d}}}-1}{2 \kappa},
$$

where $\kappa=\frac{P}{N}$ is the CSNR and $\kappa_{d}=\frac{P}{N_{d e s}}$.

In Fig. 2, we show the best power allocation for different system parameters. In our system implementations, an adjusted value of $t$ which is good over a large range of CSNRs will be chosen.

\section{System DESIGN}

1) HDA system with linear analog coding: We implement the following scheme in Fig.1. The upper part, referred to as the digital part, is formed by a VQ cascaded with a binary symmetric channel without the use of channel coding. This is realized by using hard decision decoding on the BPSK-modulated AWGN channel. Consequently, if the BPSK signals take values in $\{+1,-1\}$, the transition probabilities $\{P(j \mid i)\}$ can be obtained by $P(j \mid i)=q^{w(i, j)}(1-q)^{k-w(i, j)}$, where $w(i, j)$ denotes the Hamming distance between the binary representations of the integer $i$ and $j$, and $q$ is the crossover probability. We remark that any memoryless modulation constellation can be used besides BPSK modulation. We choose BPSK modulation because it is simple and it performs comparatively well at low CSNRs. The linear analog encoder takes the first $k$ components of the error $\mathbf{E}^{n}$ to form a scaled PAM vector $\mathbf{V}^{k}$. The linear analog decoder expands the message $\hat{\mathbf{V}}^{k}$ back to $n$ dimensions, by adding in zeros in the corresponding locations.

For a total input power $P$, a fixed power allocation $t$ and a design noise variance $N_{d e s}$, we derive an iterative training algorithm to optimize the source digital transmitter (both source encoder and source decoder) and both the digital decoder codebook and the analog decoder (as in [5]).

2) HDA system with non-linear analog coding: To improve the system performance at high CSNRs, we also implement a non-linear analog scheme which is similar to the ones studied in [3], [9]. The error vectors $\mathbf{E}^{n}$ are first quantized to some discrete values using a VQ and then mapped to a discrete set of signal points. In particular, we employ a scheme where the VQ has dimension 2 and rate $l$ bits per sample, and $2^{l}$-level PAM. For an HDA system with rate 0.5 , both components of $\mathbf{E}^{n}$ are coded using this non-linear analog coding.

The digital part employs using a channel optimized VQ (COVQ) - e.g., see [13] - which is trained with power $P_{D}=$ $(1-t) P$ and noise variance $N_{d e s}+t P$. The analog part is trained to minimized the end-to-end distortion $\left\|\mathbf{E}^{n}-\hat{\mathbf{E}}^{n}\right\|^{2}$ with power $P_{A}=t P$ and noise variance $N_{\text {des }}$.

\section{Simulation Results}

We evaluate the performance for the transmission of an i.i.d. Gaussian source over the AWGN channel. The source samples are grouped into vectors of dimension $n=24$, and transmitted with an overall rate of 0.5 channel use per source sample. We implement the two schemes proposed in Section III. Motivated by a broadcast scenario, we assume that the encoder is optimized for a given power allocation and noise variance, while the decoder knows the true noise variance and can adapt to it.

The first scheme employs linear coding in the analog part. For a fixed input power $P=1$ and design noise variance $N_{\text {des }}=0.1$ (thus $\kappa_{d}=P / N_{\text {des }}=10$ ), an iterative training algorithm as in [5] is used to generate the source digital transmitter and both the digital decoder codebook and the analog decoder. Following Lemma 2 and the curve (b) of Fig. 2, we then choose $t=0.05$.

The second scheme uses a non-linear code in the analog part. Sine the aim of this scheme is to focus on the performance at medium to high CSNRs, we assume $N_{\text {des }}=0.0001$ and $t=0.1$ ( $t$ is chosen via an experimental study). The digital part is designed using a COVQ algorithm for power $P_{D}=(1-t) P=0.9$ and noise variance 0.1001 (or around $9.5 \mathrm{~dB}$ ). The non-linear code of the analog part is designed for power $P_{A}=t P=0.1$ and noise variance 0.0001 , and 256-level PAM signals are employed.

Comparisons are made with a purely digital system and a purely analog system. The purely digital system employs solely the digital part of the HDA system, where the COVQ algorithm is used to design the source encoder. The purely analog system employs solely the analog part of the HDA system, which transmits only half of each source vectors using linear coding. Three theoretical bounds are also shown: the mismatched HDA-OPTA curve, which is given for $t=0.05$ and $\kappa_{d}=10$, the HDA-OPTA curve with $t=0.05$, and the OPTA curve, all with rate 0.5 channel use per source sample. We also compare the proposed scheme with the systems in [3], which we refer to as 'HDA-Turbo' (since the source is memoryless, the HDA-Turbo system does not employ Karhunen-Loéve processing). The digital part of the HDA-Turbo consists of a 24-dimensional 6-bit VQ (designed only for the source using the LBG algorithm) and a high-delay $(k=768, n=1536)$ rate 0.5 Turbo encoder with generator $(37,21)$ (punctured to rate 0.5$)$ and a random interleaver, and 
the analog part employs the same methods as the proposed schemes. We choose two power allocation schemes with $t=$ 0.3 and $t=0.05$.

The HDA systems and the purely digital system are trained with 300,000 vectors, and tested with 128,000 vectors. The simulation results are shown in Figs. 3 in terms of the source signal-to-distortion ratio (SDR) defined by SDR = $10 \log _{10}\left(\sigma_{s}^{2} / D\right)$, where $D$ is the MSE distortion. Overall we have the following remarks:

- The HDA schemes present a smooth and robust performance for most CSNRs, and provide substantial improvements over the purely digital COVQ system from medium to high CSNRs. They also outperform the purely analog system for a wide range of CSNRs. We also remark that the use of a linear part results in performance saturation at around $30 \mathrm{~dB}$ of CSNR.

- For the schemes with linear analog coding and $t=0.05$, the HDA scheme outperforms the HDA-Turbo systems for CSNR $\geq 7 \mathrm{~dB}$, and the HDA scheme also outperforms the HDA-Turbo with $t=0.3$ for CSNRs ranging from 15 to $40 \mathrm{~dB}$. This is due to the fact that the HDA scheme has a higher quantization rate than the HDA-Turbo systems, which results in higher saturated performance than the HDA-Turbo systems at high CSNRs. Furthermore, the HDA scheme with linear analog coding performs within 1 $\mathrm{dB}$ of the mismatched HDA-OPTA curve at high CSNRs.

- For the schemes with non-linear analog coding and $t=$ 0.1 , the HDA scheme outperforms the HDA-Turbo for CSNRs between 10 and $60 \mathrm{~dB}$. The HDA scheme still outperforms the HDA-Turbo system with $t=0.3$ at high CSNRs and saturate around $60 \mathrm{~dB}$.

In future work, we intend to compare the performance of our HDA schemes with the HDA-Turbo system studied in [3] for the transmission of Gauss-Markov sources.

\section{REFERENCES}

[1] U. Mittal and N. Phamdo, "Hybrid digital-analog (HDA) joint sourcechannel codes for broadcasting and robust communications," IEEE Trans. Inform. Theory, vol. 48, May 2002.

[2] M. Skoglund, N. Phamdo and F. Alajaji, "Design and performance of VQ-based hybrid digital-analog joint source-channel codes," IEEE Trans. Inform. Theory, vol. 48, no. 3, March 2002.

[3] M. Skoglund, N. Phamdo and F. Alajaji, "Hybrid digital-analog sourcechannel coding for bandwidth compression/expansion," submitted to IEEE Trans. Inform. Theory, May, 2005.

[4] N. Phamdo and U. Mittal, "A joint source-channel speech coder using hybrid digital-analog (HDA) modulation," IEEE Trans. Speech and Audio Processing, vol. 10, no. 4, May 2002.

[5] Y. Wang, F. Alajaji and T. Linder, "Design of VQ-based hybrid digitalanalog joint source-channel codes for image communication," Proc. IEEE Data Compression Conference, Snowbird, Utah, Mar. 2005.

[6] S. Sesia, G. Caire and G. Vivier, "Lossy transmission over slow-fading AWGN channels: a comparison of progressive, superposition and hybrid approaches," in Proc. IEEE ISIT'05, Adelaide, Australia, Sep. 2005.

[7] Z. Reznic, M. Feder and R. Zamir, "Distortion bounds for broadcasting with bandwidth expansion," submitted to IEEE Trans. Inform. Theory, Jan. 2005.

[8] J. M. Lervik, A. Grovlen and T. A. Ramstad, "Robust digital signal compression and modulation exploiting the advantages of analog communications," in Proc. IEEE GLOBECOM, Nov. 1995, pp. 1044-1048.
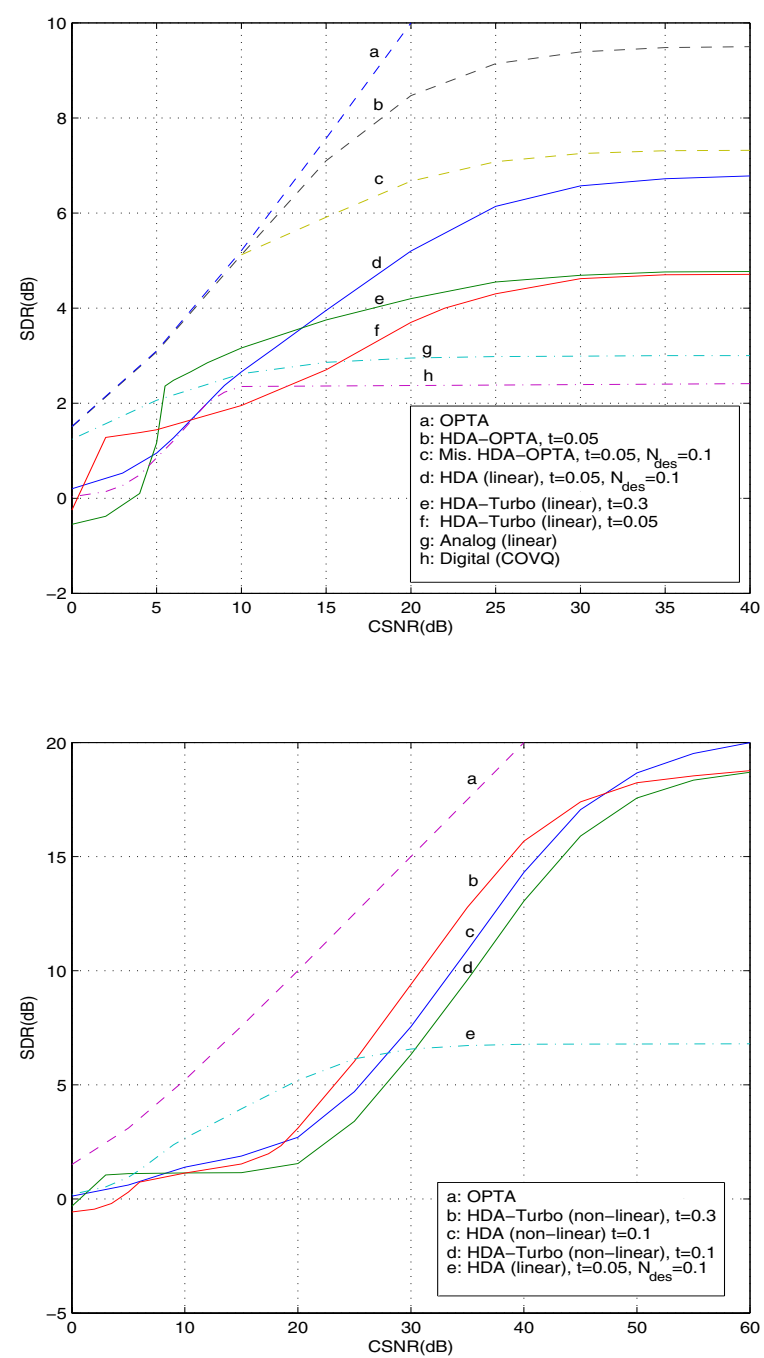

Fig. 3. Distortion performance of various HDA systems with linear (top figure) and non-linear (bottom figure) analog components

[9] A. Fuldseth and T. A. Ramstad, "Bandwidth compression for continuous amplitude channels based on vector approximation to a continuous subset of the source signal space," in Proc. IEEE ICASSP, Munich, Germany, April 1997, pp. 3093-3096.

[10] H. Coward, Joint Source-Channel Coding: Development of Methods and Utilization in Image Communications, $\mathrm{Ph} . \mathrm{D}$ thesis, Norwegian University of Science and Engineering, 2001

[11] F. Hekland, G. E. Øien, and T. A. Ramstad, "Using 2:1 Shannon mapping for joint source-channel coding," in Proc. IEEE Data Compression Conf., pp. 223-232, Mar. 2005.

[12] S. Shamai, S. Verdú and R. Zamir, "Systematic lossy source/channel coding," IEEE Trans. Inform. Theory, vol. 44, no. 2, March 1998.

[13] V. Vaishampayan, Combined Source-Channel Coding for Bandlimited Waveform Channels, Ph.D. thesis, University of Maryland, College Park, USA, 1989.

[14] V. Vaishampayan and S. I. R. Costa, "Curves on a sphere, shiftmap dynamics, and error control for contiuous alphabet sources," IEEE Trans.Inform. Theory, vol. 49, no. 7, July 2003.

[15] C. E. Shannon, "A mathematical theory of communication," Bell Syst. Tech. J., vol. 27, pp. 379-423 and 623-656, 1948. 